\title{
基于 $\mathrm{t}$ 分布邻域嵌入与 XGBoost 的刀具 多工况磨损评估 ${ }^{*}$
}

\author{
李 亚 黄亦翔 赵路杰 刘成良 \\ (上海交通大学机械系统与振动国家重点实验室 上海 200240)
}

\begin{abstract}
摘要: 刀具磨损的在线检测是未来自动化生产的必备功能, 良好的评估模型可以有效地提高加工质量, 降低经济损失。在现 有研究基础上, 提出了一种优化的刀具磨损评估方法, 该方法综合使用了刀具切削过程中主轴的电流信号与振动信号, 改善 了单信号分析的不足。对采集到的信号提取时域、频域以及小波包特征，尽可能全面地提取了信号的有效信息。采用 $\mathrm{t}$ 分布 邻域嵌入算法对特征进行降维, 并使用 K-means 算法将多种不同的工况根据特征进行聚类, 进一步提高了模型预测的准确率 和泛化能力。最后使用 XGBoost 集成学习算法作为评估器, 采用回归和分类两种方式对该模型进行评价。结果表明, 样本不 平衡问题对 XGBoost 算法的影响较小, 和随机森林等传统集成学习算法相比, XGBoost 在预测精度上有明显提升, 在预测时 间上减少一个数量级, 是一种更为准确高效的刀具磨损检测算法, 可以广泛地在工业上进行应用。
\end{abstract}

关键词: 刀具磨损; 健康评估; $\mathrm{t}$ 分布邻域嵌入; XGBoost

中图分类号: TH17

\section{Multi-condition Wear Evaluation of Tool Based on T-SNE and XGBoost}

\author{
LI Ya HUANG Yixiang ZHAO Lujie LIU Chengliang
}

(State Key Laboratory of Mechanical System and Vibration, Shanghai Jiao Tong University, Shanghai 200240)

\begin{abstract}
On-line inspection of tool wear is a necessary function for future automated production. A good evaluation model can effectively improve machining quality and reduce economic loss. Based on the existing research, an optimized tool wear evaluation method is presented. Current signal and vibration signal of spindle in cutting process are used synthetically, which can effectively improve the deficiency of single signal analysis. Time domain, frequency domain and wavelet packet features are extracted from the collected signals, and the signal feature information is extracted as comprehensively as possible. T-SNE is used to reduce the dimension of features, and K-means is used to cluster various working conditions according to the similarity of features, which further improves the accuracy and generalization ability of model prediction. Finally, the integrated learning algorithm XGBoost is used as the estimator, and the model is evaluated by regression and classification. The results show that the problem of sample imbalance has less impact on the XGBoost algorithm. Compared with the traditional integrated learning algorithms such as random forest, XGBoost improves the prediction accuracy and reduces the prediction time by an order of magnitude. It is a more accurate and efficient tool detection algorithm and can be widely used in industry.
\end{abstract}

Key words: tool wear; health assessment; t-SNE; XGBoost

\section{0 前言}

在数控机床的生产制造过程中, 刀具状态的检 测是一个极为重要的课题。一方面过度磨损的刀具 性能会发生改变, 不但会影响加工的质量, 还会产 生刀具破损, 研究表明, 约 20\%的停机事件与刀具 失效有关 ${ }^{[1]}$; 另一方面, 刀具状态的检测也是未来

* 国家重点研发计划(2017YFB1302004)与国家自然科学基金(51975356) 资助项目。20190314 收到初稿, 20190810 收到修改稿
无人化、自动化生产的必要环节。在目前的研究中, 刀具磨损的检测按照原理可以分为直接法和间接 法。直接法是指直接对刀具的几何尺寸、磨损状态 等进行测量, 直接测量有机械测量、电阻测量、射 线测量等方法, 常见的是采用图像识别的手段。SU 等 ${ }^{[2]}$ 利用 CCD 相机等设备来获取刀具的图像, 使用 边缘检测的方式计算出刀面的磨损值; LI 等 ${ }^{[3]}$ 通过 机器视觉的方式获取刀具图像, 利用灰度共生矩阵 生成纹理特征, 通过度量纹理的熵实现对刀具磨损 状态的评估。这些与视觉相关的测量方式布置难度 
较大, 容易受到切屑、冷却液的影响, 在处理速 度上也较慢。间接测量是指通过检测切削力 ${ }^{[4]}$ 、电 流、振动、声发射信号, 或者工件表面的加工状 况, 来评估刀具磨损的方法。 RAO 等 ${ }^{[5]}$ 使用激光 多普勒测振仪采集铣削加工中的信号, 提取时频 特征后通过人工神经网络建立刀具磨损的评估模 型; 王丽华等 ${ }^{[6]}$ 通过采集加工过程中的声发射信号, 采用堆叠降噪自编码器实现对刀具磨损量的评估;

NIAKI 等 ${ }^{[7]}$ 采集主轴电流的信息, 通过卡尔曼滤波 的方式预测刀具的磨损量。这些研究的不足在于只 是针对某一种信号进行研究, 而且采用的算法往往 需要足够多的样本作为支撑。事实上, 不同的信号 具有不同的特性, 振动、声发射信号可以准确记录 加工过程的全部信息, 但是易受到噪声的干扰, 电 流信号可以通过计算主轴功率间接反映切削力的变 化, 但是灵敏度不如振动信号。另外, 在间接测量 的研究中很少有针对多工况下刀具的磨损情况进行 研究的, 而不同工况下刀具的磨损的情况是不同的。

对于上述研究中存在的一些不足和实际中可能 存在的一些问题 ${ }^{[8-12]}$, 本文提出以下改进点。针对 单一信号的缺陷, 本文综合采用了电流信号与振动 信号, 力求实现两种信号的优势互补; 针对实际加 工中多工况的问题, 采用了 $\mathrm{t}$ 分布邻域嵌入 (t-SNE) 算法对特征进行降维, 再使用 K-means 算法将多种 工况进行聚类, 一定程度上提高了预测的准确率; 针对样本不平衡的问题, 集成学习往往有较好的效 果, 本文采用极限梯度提升 (Extreme gradient boosting, XGBoost)作为评估器并和其他传统集成学 习的方法进行对比, 试验表明, XGBoost 在预测精 度上要优于其他集成学习方法, 在运算时间上要小 一个数量级。

\section{1 刀具磨损评估原理}

\section{1 刀具磨损过程}

在切削过程中, 刀具的切削部分与工件表面、切 屑产生剧烈的摩擦, 产生巨大的压力和很高的温度, 结果就会使刀具磨针, 逐渐失效。刀具的磨损与机械、 热和化学密切相关, 一般可分为正常磨损和非正常磨 损, 本文研究的对象是正常磨损的刀具。正常的刀具 磨损存在一个渐变的过程 ${ }^{[8]}$, 一般可以分为三个阶段, 如图 1 所示: 初期磨损、正常磨损和急剧磨损。新刀 具刃口锋利、表面粗粘, 后刀面与加工表面的接触面 积小、应力大, 很容易就在后刀面产生磨损, 故初期 磨损的斜率较大; 到正常磨损阶段, 刀具的磨损比较 均匀, 后刀面的磨损量与切削时间近似呈线性关系, 这个阶段是刀具的有效工作阶段; 当后刀面磨损增大
到一定程度后, 加工表面的粗糙值变大, 切应力随之 增大、切削温度升高, 刀具磨损急剧加快。影响刀具 磨损速率的因素有工件材质、刀具材质、加工工况等 诸多因素, 本文将针对切削深度、进给速度、切削速 度三个因素进行研究。

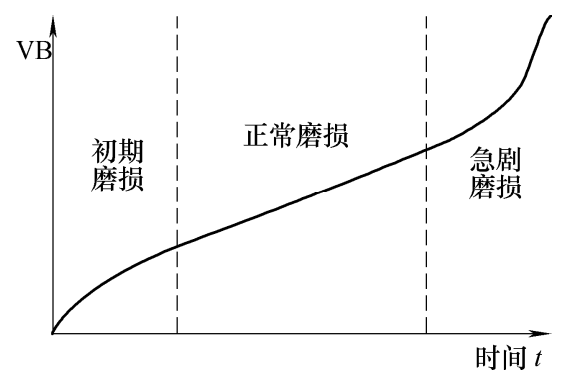

图 1 刀具的磨损曲线

\section{2 主轴电流与刀具磨损关系}

相同工况下随着刀具的磨损, 加工所产生的切削 力也将产生变化, 切削力的变化通过刀柄、主轴等传 递到主轴电动机, 进而引起电动机电流的变化, 其关 系方程如下

$$
J \frac{\mathrm{d} \omega}{\mathrm{d} t}=K I_{r m s}-B \omega-F R
$$

式中, $J$ 为转动部件的总惯量; $\omega$ 为主轴角速度; $K$ 为电动机扭矩常量; $I_{r m s}$ 为电动机的有效值; $B$ 为 黏性阻尼系数; $F$ 为切削力; $R$ 为刀具的半径 $(F \cdot R$ 即为切削力矩)。

本文中试验所用机床的主轴电动机为三相异步 交流电动机, 故分别测出三相电流, 采用均方根法 计算出电流的有效值

$$
I_{r m s}=\sqrt{\frac{\left(i_{u}^{2}+i_{v}^{2}+i_{w}^{2}\right)}{3}}
$$

式中, $i_{u} 、 i_{v} 、 i_{w}$ 代表电动机的三相电流。

单次试验中工况恒定, 主轴转速 $\omega$ 一般不发生 变化, 故 $\mathrm{d} \omega / \mathrm{d} t$ 为零, 而 $K 、 R 、 B 、 \omega$ 为 定值, 所以在不考虑外界干扰的情况下, 根据(1)式 可知主轴电流与切削力在理论上存在某种确定的关 系, 故通过测量主轴电流间接分析刀具的磨损情况 在理论上是可行的, 电流信号随刀具磨损的变化示 意图如图 2 所示。

\section{3 信号采集流程}

从数控机床采集的信号分为振动信号和电流信号。 振动传感器安装在主轴靠近端面处, 可以直接记录 加工过程所产生的各种细节; 电流传感器采集的是 主轴的电流信号, 通过机床内置传感器获取。采集 的原始信号接入数据采集卡, 经放大和滤波后传入 电脑, 电脑端使用的采集软件是 LabVIEW。 


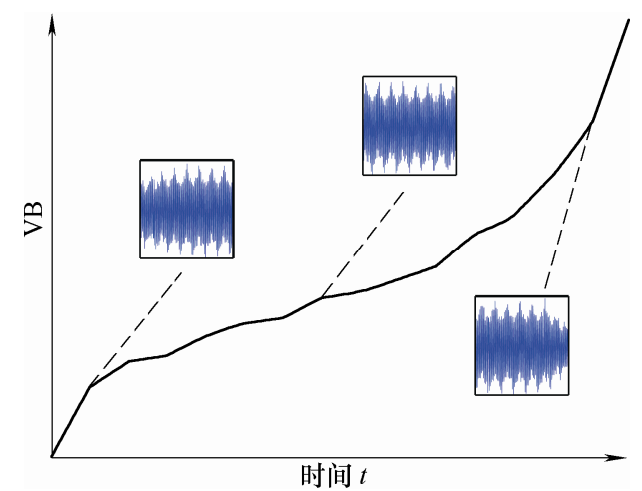

图 2 电流信号随刀具磨损逐渐变化

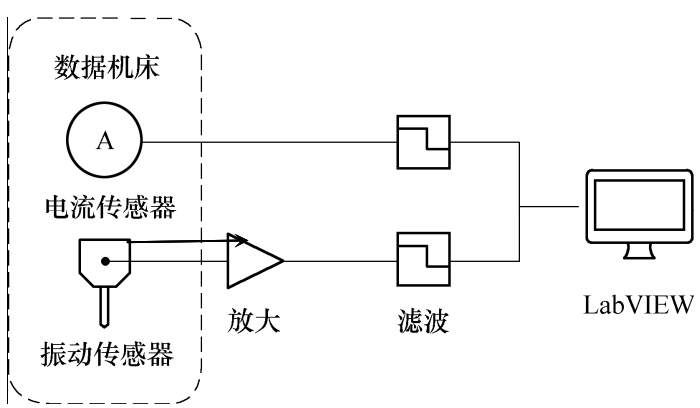

图 3 数据采集流程

\section{4 数据分析流程}

数据分析的整个流程如图 4 所示。首先将原 始数据进行预处理, 预处理包括异常值、缺失值 的处理以及数据的分割, 数据分割的目的是将有 效工作信号和空载信号分割开来; 之后利用常规 信号分析的方法从数据中提取特征, 包括时域特 征、频域特征和小波包特征; 之后将特征归一化， 采用 t-SNE 和 K-means 算法进行降维分类, 最后 将特征和分类结果输入 XGBoost 的模型进行训 练, 给出回归和分类的预测结果。在降维分类的 部分, 将对比 t-SNE、PCA 以及自编码器三种常 见的降维手段; 在模型训练部分, 将对比 GBDT、 Random Forest、AdaBoost 以及 XGBoost 四种集成 学习的方法预测效果。

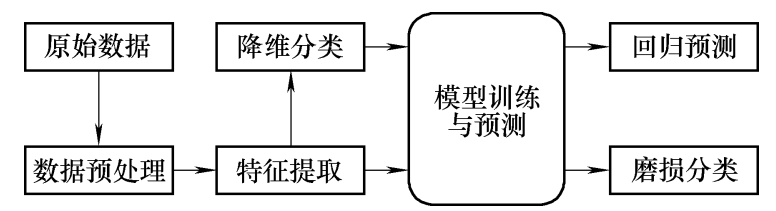

图 4 数据处理流程

\section{2 刀具磨损预测方法}

\section{$2.1 \quad t-S N E$}

t-SNE 是由 SNE 发展而来的一种非线性的降 维方法, 适合用于将高维的数据降到 2 维或者 3
维, 并进行可视化的显示 ${ }^{[9]}$ 。和其他一些降维手 段相比, 其优势在于既可以捕捉到高维数据的局 部结构, 也可以保留一些全局的结构, 所以能直 观地将数据进行分类。t-SNE 的核心思想在于将欧 氏距离概率化，利用联合概率计算数据之间的相 似性, 再通过最小化 KL 散度(Kullback-Leibler divergence)实现低维分布与高维分布的高相似性。 其基本的步骤如下。

设 $m$ 个处在高维的数据为 $\left\{x_{1}, x_{2}, \cdots, x_{m}\right\}$, 其两 两之间的相似条件概率可以表示为

$$
p_{j \mid i}=\frac{\exp \left(-\left\|x_{i}-x_{j}\right\|^{2} / 2 \sigma_{i}^{2}\right)}{\sum_{k \neq i} \exp \left(-\left\|x_{i}-x_{k}\right\|^{2} / 2 \sigma_{i}^{2}\right)}
$$

$\sigma_{i}$ 是以 $x_{i}$ 为中心的高斯函数的方差。这样就可 以把高维空间中任意两个数据点的联合分布概率表 示为

$$
p_{i j}=\frac{p_{i \mid j}+p_{j \mid i}}{2 m}
$$

在低维空间中, 用 $m$ 个数据 $\left\{y_{1}, y_{2}, \cdots, y_{m}\right\}$ 来表 示高维数据的分布, 采用具有长尾特性的 $\mathrm{t}$ 分布(自 由度为 1), 则低维数据两两之间的相似条件概率可 以表示为

$$
q_{i j}=\frac{\left(1+\left\|y_{i}-y_{j}\right\|^{2}\right)^{-1}}{\sum_{k \neq l}\left(1+\left\|y_{k}-y_{l}\right\|^{2}\right)^{-1}}
$$

低维数据与高维数据之间的 KL 散度表示为

$$
C=K L(P \| Q)=\sum_{i} \sum_{j} p_{i j} \log _{2} \frac{p_{i j}}{q_{i j}}
$$

优化 KL 散度的梯度如下

$$
\frac{\delta c}{\delta y_{i}}=4 \sum_{j}\left(p_{i j}-q_{i j}\right)\left(y_{i}-y_{j}\right)\left(1+\left\|y_{i}-y_{j}\right\|^{2}\right)^{-1}
$$

下面来确定 $\sigma_{i}$, 不同的 $x_{i}$ 具有不同的 $\sigma_{i}, \mathrm{t}-\mathrm{SNE}$ 中使用复杂度参数来定义高斯函数的形态, 复杂度 可以理解为 $x_{i}$ 临近的有效点的度量。定义 $P_{i}$ 表示给 定 $x_{i}$ 下其他所有数据点的条件概率分布, 则复杂度 的定义为

$$
P\left(P_{i}\right)=2^{H\left(P_{i}\right)}
$$

其中, $H\left(P_{i}\right)$ 表示 $P_{i}$ 的熵

$$
H\left(P_{i}\right)=-\sum_{j} p_{j \mid i} \log _{2} p_{j \mid i}
$$

给定复杂度之后, 采用二分搜索的方式寻找出 合适的 $\sigma_{i}$, 复杂度通常可以选择在 5 50 之间。

t-SNE 中使用的是概率化的欧氏距离, 其优势 
在于可以直观地进行分类和判断。

\subsection{XGBoost}

XGBoost 是一种由梯度提升决策树(Gradient boosting decision tree, GBDT)发展而来的算法 ${ }^{[10]}$, 其本质仍在于梯度提升, 但和传统的一些算法相比, 其创新性地引入了二阶导数和正则项, 加之系统层 面的一些优化，使得该算法不但训练效果好，而且 运算速度也很快, 在近些年的各种数据竞赛中大放 异彩, 并被广泛应用于各个领域。其基本步骤如下。

有 $n$ 个训练数据 $\left\{\left(x_{1}, y_{1}\right),\left(x_{2}, y_{2}\right) \cdots\left(x_{n}, y_{n}\right)\right\}, K$ 棵树 $\left\{f_{1}, f_{2}, \cdots, f_{K}\right\}$, 则数据 $x_{i}$ 的预测值为

$$
\hat{y}_{i}=\sum_{k=1}^{K} f_{k}\left(x_{i}\right)
$$

训练的目标函数为

$$
\mathcal{L}=\sum_{i=1}^{n} l\left(y_{i}, \hat{y}_{i}\right)+\sum_{k=1}^{K} \Omega\left(f_{k}\right)
$$

其中第一项是训练误差, 用于衡量模型与数据的匹 配程度，第二项是正则项，用于衡量模型的复杂度， 具体表达式如下

$$
\begin{gathered}
l\left(y_{i}, \hat{y}_{i}\right)=\left(y_{i}-\hat{y}_{i}\right)^{2} \\
\Omega\left(f_{k}\right)=\gamma T+\frac{1}{2} \lambda \sum_{j=1}^{T} w_{j}^{2}
\end{gathered}
$$

式中, $T$ 表示 $f_{k}$ 叶节点的个数; $w_{j}$ 表示第 $j$ 个叶节 点的权值; $\gamma$ 和 $\lambda$ 为伸缩系数。

对于第 $t$ 棵树有

$$
\begin{gathered}
\mathcal{L}^{(t)}=\sum_{i=1}^{n} l\left(y_{i}, \hat{y}_{i}^{(t)}\right)+\sum_{i=1}^{t} \Omega\left(f_{i}\right)= \\
\sum_{i=1}^{n} l\left(y_{i}, \hat{y}_{i}^{(t-1)}+f_{t}\left(x_{i}\right)\right)+\Omega\left(f_{t}\right)+\text { constant }
\end{gathered}
$$

利用二阶泰勒展开, 并设 $g_{i}=\partial_{\hat{y}^{(t-1)}} l\left(y_{i}, \hat{y}^{(t-1)}\right)$ 和 $\hat{h}_{i}=\partial_{\hat{y}^{(t-1)}}^{2} l\left(y_{i}, \hat{y}^{(t-1)}\right)$, 有

$$
\begin{gathered}
\mathcal{L}^{(t)} \approx \sum_{i=1}^{n}\left[l\left(y_{i}, \hat{y}_{i}^{(t-1)}\right)+g_{i} f_{t}\left(x_{i}\right)+\frac{1}{2} h_{i} f_{t}^{2}\left(x_{i}\right)\right]+ \\
\Omega\left(f_{t}\right)+\text { constant }= \\
\sum_{i=1}^{n}\left[g_{i} f_{t}\left(x_{i}\right)+\frac{1}{2} h_{i} f_{t}^{2}\left(x_{i}\right)\right]+\Omega\left(f_{t}\right)+ \\
{\left[\sum_{i=1}^{n} l\left(y_{i}, \hat{y}_{i}^{(t-1)}\right)+\text { constant }\right]}
\end{gathered}
$$

式中, $\left[\sum_{i=1}^{n} l\left(y_{i}, \hat{y}_{i}^{(t-1)}\right)+\right.$ constant $]$ 与最优值无关, 可以直接舍去, 同时定义 $q\left(x_{i}\right)$ 表示 $x_{i}$ 所属树叶的 位置, $I_{j}=\left\{i \mid q\left(x_{i}\right)=j\right\}$ 表示第 $j$ 个叶节点中样本
序号的集合，则有

$$
\begin{aligned}
\widetilde{\mathcal{L}}^{(t)} \approx & \sum_{i=1}^{n}\left[g_{i} f_{t}\left(x_{i}\right)+\frac{1}{2} h_{i} f_{t}^{2}\left(x_{i}\right)\right]+\gamma T+\frac{1}{2} \lambda \sum_{j=1}^{T} w_{j}^{2}= \\
& \sum_{j=1}^{T}\left[\left(\sum_{i \in I_{j}} g_{i}\right) w_{j}+\frac{1}{2}\left(\sum_{i \in I_{j}} h_{i}+\lambda\right) w_{j}^{2}\right]+\gamma T
\end{aligned}
$$

假设树的结构已经确定，那么最优权值为

$$
w_{j}^{*}=-\frac{\sum_{i \in I_{j}} g_{i}}{\sum_{i \in I_{j}} h_{i}+\lambda}
$$

设 $G_{j}=\sum_{i \in I_{j}} g_{i}, H_{j}=\sum_{i \in I_{j}} h_{i}$, 则

$$
\widetilde{\mathcal{L}}^{(t)}(q)=-\frac{1}{2} \sum_{j=1}^{T} \frac{G_{j}^{2}}{H_{j}+\lambda}+\gamma T
$$

由此可以计算一个节点分裂前后的差值

$$
P=\mathcal{L}_{\text {entire }}-\mathcal{L}_{\text {split }}=
$$

$$
\begin{gathered}
\frac{1}{2}\left[\frac{G_{L}^{2}}{H_{L}+\lambda}+\frac{G_{R}^{2}}{H_{R}+\lambda}-\frac{\left(G_{L}+G_{R}\right)^{2}}{H_{L}+H_{R}+\lambda}\right]+ \\
\gamma\left(T_{\text {entire }}-T_{\text {split }}\right)
\end{gathered}
$$

式中, $G_{L}, H_{L}$ 为左枝对应的参数; $G_{R}, H_{R}$ 为右枝对 应的参数。之后就可以从树深为 0 开始不断遍历所 有特征进行建树, 直到 $P$ 为负时停止; 或者先将树 分裂到最大深度, 再依据上式将所有 $P$ 为负的树枝 进行裁剪, 从而得到最优的模型。

\section{3 试验方案}

试验方案采用全因子试验设计，使用的机床型 号为 $\mu 1000 / 460 \mathrm{VF}$ ，切削刀具使用的是金路 UA100-S3-10025 型铜铝合金通用立铣刀, 铣刀有三 刃, 螺旋角为 $45^{\circ}$, 刃径 $10 \mathrm{~mm}$, 加工工件为铝合 金, 机床的控制系统是华中数控 HNC-818B。振动 传感器型号为标智 bw J14530, 振动采集卡为 NI 9234, 振动传感器固定在主轴上靠近端面的地方, 电流信号利用华中数控的二次开发接口以 IP 协议 的方式从机床直接读取。

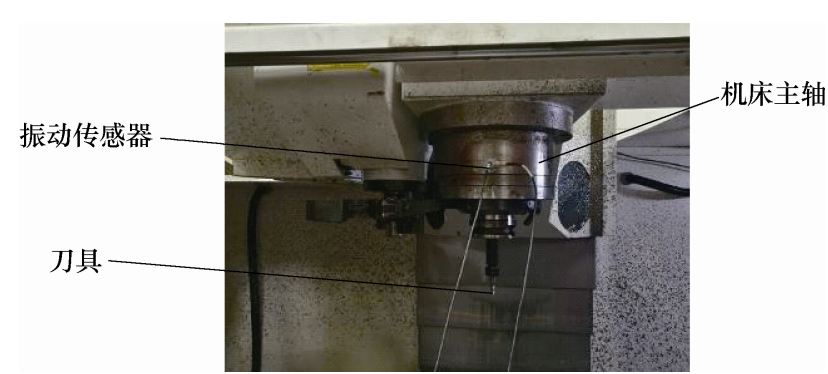

图 5 传感器布置 
工件材质为铝合金, 切削深度、进给速度和切 削速度为三个可变因子, 切削深度为 $1 \mathrm{~mm}$ 和 0.5 $\mathrm{mm}$, 进给速度为 $0.5 \mathrm{~mm} / \mathrm{r}$ 和 $0.25 \mathrm{~mm} / \mathrm{r}$, 切削速度 为 $50 \mathrm{~m} / \mathrm{min}$ 和 $100 \mathrm{~m} / \mathrm{min}$, 组合共 8 种工况, 具体 方案如表 1 所示。

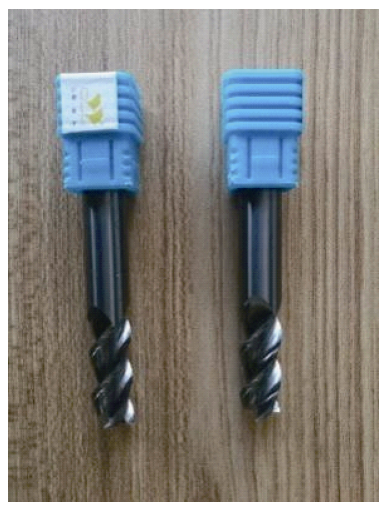

图 6 试验刀具

表 1 试验方案表

\begin{tabular}{cccc}
\hline 工况 & 切削深度 $/ \mathrm{mm}$ & 进给速度 $/(\mathrm{mm} / \mathrm{r})$ & 切削速度 $/(\mathrm{m} / \mathrm{min})$ \\
\hline 1 & 1.0 & 0.50 & 50 \\
2 & 0.5 & 0.50 & 50 \\
3 & 0.5 & 0.25 & 50 \\
4 & 1.0 & 0.25 & 50 \\
5 & 1.0 & 0.50 & 100 \\
6 & 1.0 & 0.25 & 100 \\
7 & 0.5 & 0.25 & 100 \\
8 & 0.5 & 0.50 & 100 \\
\hline
\end{tabular}

每次试验取全新刀具进行全生命周期试验, 振 动信号采样频率为 $10 \mathrm{kHz}$, 电流信号采样频率为 1 $\mathrm{kHz}$, 采样间隔为 $10 \mathrm{~min}$, 采样时长为 $10 \mathrm{~s}$, 每次 采样后测量并记录刀具的后刀面磨损量(VB 值), 当 VB 值超出阈值后试验结束。

\section{4 数据处理}

\section{1 数据预处理}

(1) 数据清洗。

无论是训练集还是测试集都会存在一些异常数 据, 异常数据会给模型预测带来较大的偏差, 在进 行数据分析前根据以下原则对异常值进行处理: 对 单个的异常值或缺失值以插值法进行处理, 对于连 续的异常数据予以删除。

(2) 数据分割。

在信号采集过程中刀具除了有正常切削状态, 还可能会有空转、接触工件、离开工件等过程, 不 同阶段所包含的有效信息不同, 正常切削和接触工 件阶段包含的有效信息较多, 空载和离开工件阶段 包含的有效信息较少, 因此需要对采集到的信号进 行状态划分, 选取包含有效信息的信号进行分析。

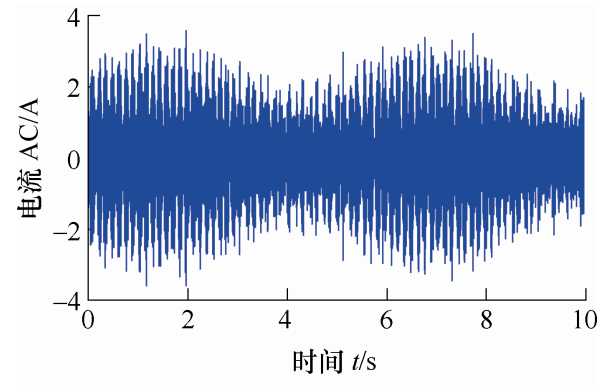

(a) 正常切削阶段信号

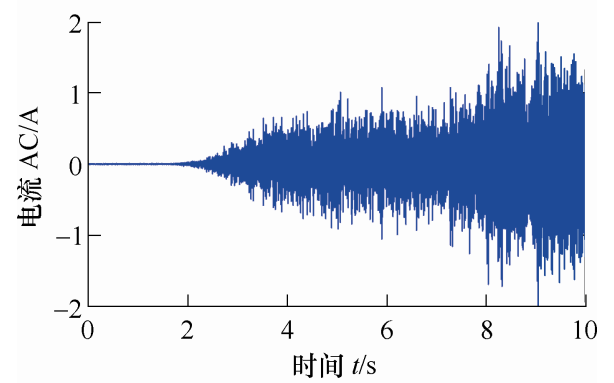

(b) 接触工件阶段信号

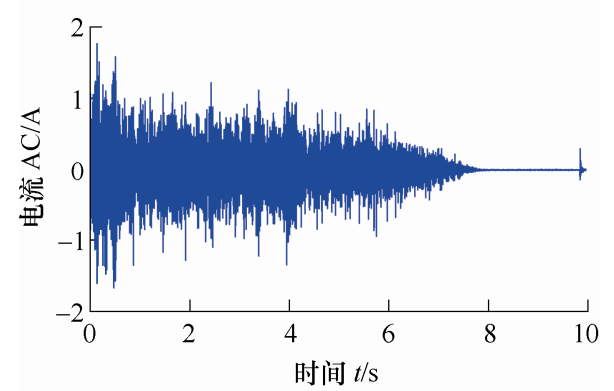

(c) 离开工件阶段信号

图 7 典型信号示例

一个完整的走刀过程可以分为四个阶段：空载 阶段、上升阶段(刀具与工件开始接触)、平稳阶段(正 常切削)、下降阶段(刀具离开工件)。阶段划分的方 法为: 根据原始信号计算幅值随时间的变化, 将幅 值信号平滑处理后计算一阶差分，差分大于某个阈 值的部分即是上升阶段, 小于某个阈值的部分即是 下降阶段，正常切削阶段处于上升和下降部分之间。

\section{2 特征提取}

在特征提取时, 根据特征的类型可以分为三部 分：时域特征、频域特征和小波包特征。

(1) 时域特征。

时域的特征主要是针对时域的波形进行分析, 时域 分析的意义在于可以有效的提取对磨损量比较敏感的特 征，如直流分量的最大值、交流分量的有效值等。时域 特征包括有量纲的参数: 均值、有效值、歪度、峭度等 和量纲-参数: 脉冲指标、裕度指标、峭度指标等。

(2) 频域特征。

频域分析也是信号分析中常有的手段, 一般采 用傅里叶变换的方式将时域信号转换为频域信号, 然后再进行图谱分析和特征参数的提取, 频域分析 的意义在于刀具产生磨损后会改变频谱中频率的分 
布, 进而引起重心频率、峰值频率等特征的变化。 记信号的频率谱为 $p(f)$, 功率谱为 $S(f)$, 则部分 频域参数的计算公式如表 2 所示。

表 2 频域特征计算公式

\begin{tabular}{|c|c|c|c|}
\hline 特征 & 计算式 & 特征 & 计算式 \\
\hline $\begin{array}{l}\text { 频率 } \\
\text { 均方 }\end{array}$ & $\mathrm{MSF}=\frac{\sum_{i=0}^{n} f_{i}^{2} S\left(f_{i}\right)}{\sum_{i=0}^{n} S\left(f_{i}\right)}$ & $\begin{array}{c}\text { 频率均 } \\
\text { 方根 }\end{array}$ & $\mathrm{RMSF}=\sqrt{\mathrm{MSF}}$ \\
\hline $\begin{array}{l}\text { 频率 } \\
\text { 重心 }\end{array}$ & $\mathrm{FC}=\frac{\sum_{i=0}^{n} f_{i} S\left(f_{i}\right)}{\sum_{i=0}^{n} S\left(f_{i}\right)}$ & $\begin{array}{c}\text { 频率标 } \\
\text { 准差 }\end{array}$ & $\mathrm{RVF}=\sqrt{\mathrm{VF}}$ \\
\hline $\begin{array}{l}\text { 频率 } \\
\text { 方差 }\end{array}$ & $\mathrm{VF}=\frac{\sum_{i=0}^{n}\left(f_{i}-F C\right)^{2} S\left(f_{i}\right)}{\sum_{i=0}^{n} S\left(f_{i}\right)}$ & $\begin{array}{l}\text { 峰值 } \\
\text { 频率 }\end{array}$ & $F_{m}=\operatorname{argmax}(p(f))$ \\
\hline
\end{tabular}

(3) 小波包特征。

小波包特征提取的意义在于弥补频域分析的 不足, 由于傅里叶变换的窗口固定, 一些非平稳 的、短时的特征无法直接体现, 采用小波包分解 可以得到多个频带, 相关度较高的频带特征在后 续处理中可以有效地与其他频带区别开来, 能有 效减少噪声带来的影响。本试验采用的是 “db6” 小波包进行 5 层分解, 对子频带提取均值、最值、 峭度等特征。

\section{5 工况分类}

试验共有 8 种工况, 如果直接将全部数据放入 模型进行训练, 容易产生工况间的混淆, 降低模型 预测的准确率; 如果是对每种工况单独进行预测, 由于样本数量较小, 也容易引起偏差, 且单工况预 测的模型泛化能力较差。一个折中的方法是采用聚 类的方法对相似的工况进行聚合, 再对每一个合集 训练出对应的模型参数, 当有新的工况加入时, 可 以根据工况间的相似性划分入对应的合集中, 然后 再进行预测。

对工况进行聚类, 理想的聚类结果是同一工况 的样本点距离较近, 不同工况的样本点或是重叠(说 明是相似工况)或是相距很远(不相似工况)。由于特 征维度较高, 一般采用降维加聚类的方式。传统的 降维方法以 PCA 应用最为广泛, 由深度学习所衍生 的自编码器等降维手段也具有不错的效果, t-SNE 则是由 SNE 发展而来的一种降维算法, 在聚类与可 视化问题上都有很好的表现。下面以前 4 种工况为 例, 分别使用 t-SNE、PCA 和自编码器三种降维方
式对数据进行处理, 然后采用 K-means 算法聚类, 最后采用调整兰德系数(Adjusted rand index, ARI) 评价聚类结果的优劣, 结果如图 8(图中虚线为 K-means 分类边界)。不同分类结果的 ARI 见表 3 。

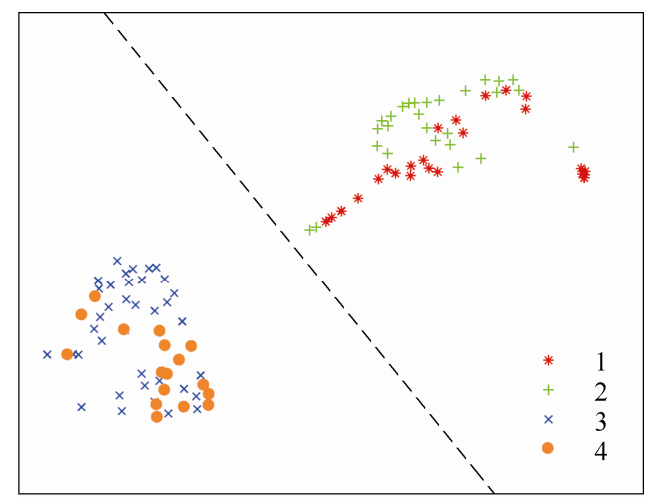

(a) t-SNE 降维分类结果

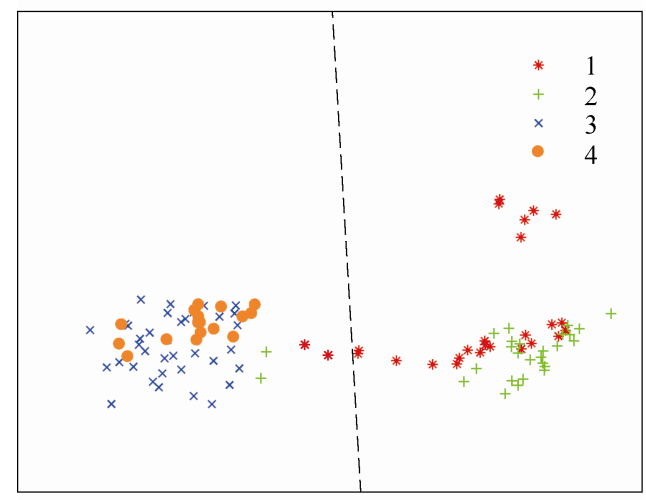

(b) PCA 降维分类结果

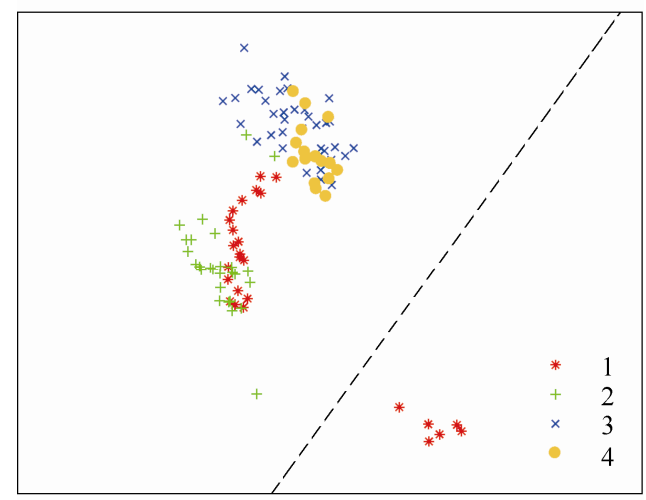

(c) 自编码器降维分类结果

图 8 不同降维方法的分类结果

表 3 不同分类结果的 ARI

\begin{tabular}{cccc}
\hline 方法 & t-SNE & PCA & BM \\
\hline ARI & 0.528 & 0.454 & 0.019 \\
\hline
\end{tabular}

可以看出, 采用 $\mathrm{t}-\mathrm{SNE}$ 降维之后可以准确地将 数据分为两类, 采用 PCA 可以大致将数据分为两 类, 但部分数据分类错误; 采用自编码器在分类之 后则出现了明显的偏差。可见, 在工况聚类的问题 上采用 t-SNE 降维可以取得不错的效果, 同样采用 t-SNE 对后 4 种工况的分类结果如图 9 所示。 


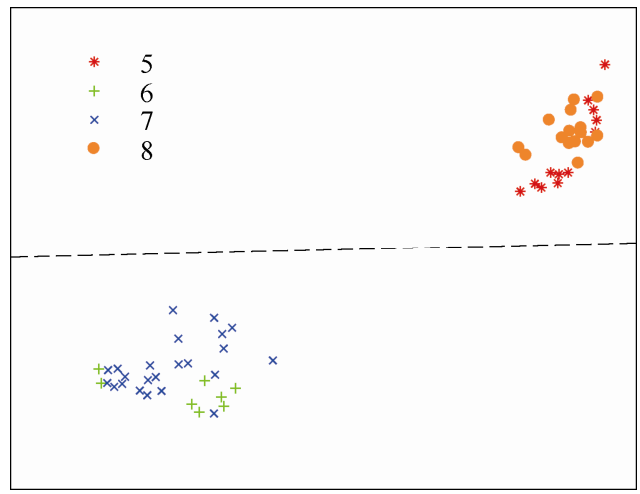

图 9 其他工况分类结果

由上, 可以将原来的 8 种单一工况聚合为 4 组 相似工况，如表 4 所示。

表 4 工况分组结果

\begin{tabular}{ccc}
\hline 组别 & 工况 & 工件材质 \\
\hline I & $1 \& 2$ & \\
II & $3 \& 4$ & 铝合金 \\
III & $5 \& 8$ & \\
IV & $6 \& 7$ & \\
\hline
\end{tabular}

\section{6 模型评估}

\section{1 回归预测}

对于刀具磨损问题，其样本的典型特征是各个 阶段分布不均衡, 对于这样的样本, 集成学习往往 有不错的效果。传统的集成学习方法有 Random Forest, AdaBoost, GBDT 等, 而 XGBoost 则是近 年来兴起的集成学习方法, 在效率和性能方面均有 不错的提升。下面将采用上述若干集成学习的方法 对刀具磨损进行评估, 并比较其结果。

首先使用回归预测的方法进行试验, 以刀具的 $\mathrm{VB}$ 值作为标签, 采用平均绝对误差作为损失函数。 为了减少偶然误差, 试验共进行 10 次, 每次试验先 对数据集随机进行划分, 然后分别采用不同的算法 对聚类得到的 4 组工况进行训练和预测, 记录预测 的平均绝对误差和用时, 最后对 10 组试验的结果取 平均值, 结果如图 10、11 所示。

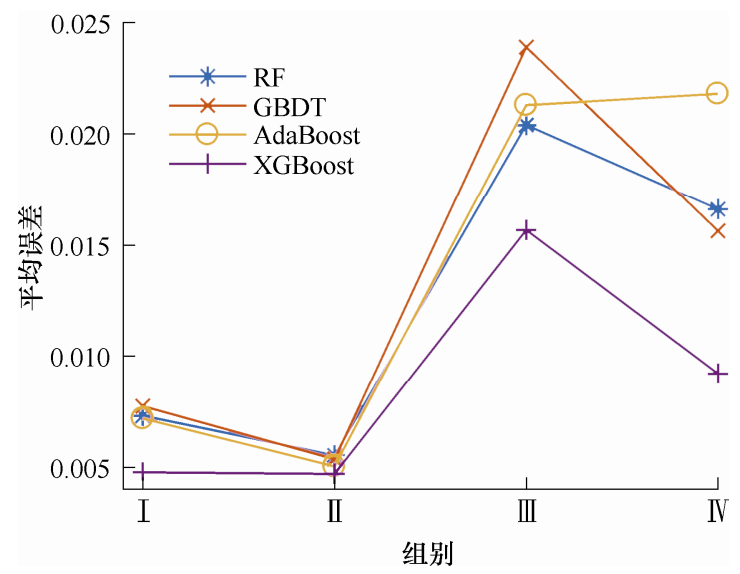

图 10 不同评估器平均误差对比

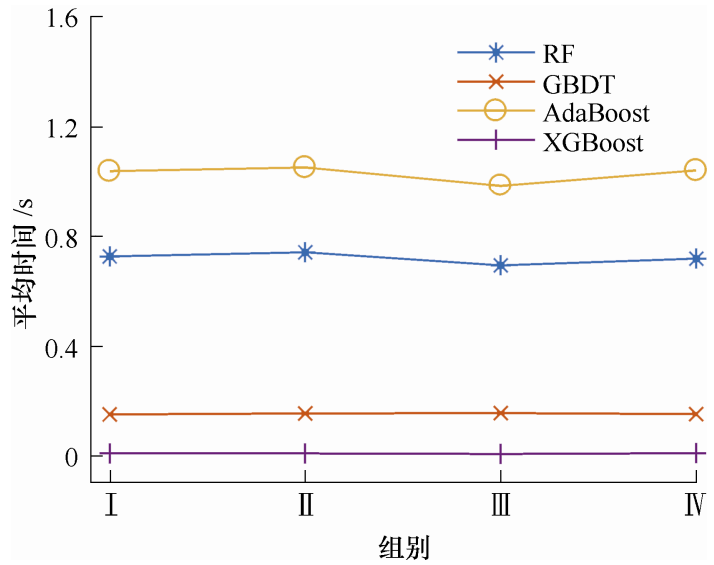

图 11 不同评估器用时对比

表 5 不同评估器评估结果

\begin{tabular}{ccccc}
\hline 方法 & $\begin{array}{c}\text { Random } \\
\text { Forest }\end{array}$ & GBDT & AdaBoost & XGBoost \\
\hline 加权平均误差 & 0.0106 & 0.0111 & 0.0115 & 0.0074 \\
总用时/s & 2.8756 & 0.6069 & 4.1138 & 0.0311 \\
\hline
\end{tabular}

从平均误差来看，对于工况 I 和 II，所有的方 法误差不大，对于工况 III 和 IV，XGBoost 相比其 他方法误差有显著的减小; 从运行的时间上来看, XGBoost 的平均运行时间不到 $0.01 \mathrm{~s}$, 相比 GBDT 要小一个数量级, 对比 Random Forest 和 AdaBoost 要小两个数量级。不难看出, 在刀具 VB 值预测的 问题上，XGBoost 相较传统方法在精度上有明显提 升，在计算速度上快至少一个数量级。

在第 5.1 节中提到三种训练的方式, 一种是对全 部数据进行训练预测, 一种是对每个工况单独进行 训练预测, 一种是如本节所述, 先对工况进行聚类, 再训练预测, 这三种方式的平均绝对误差如图 12 所示。

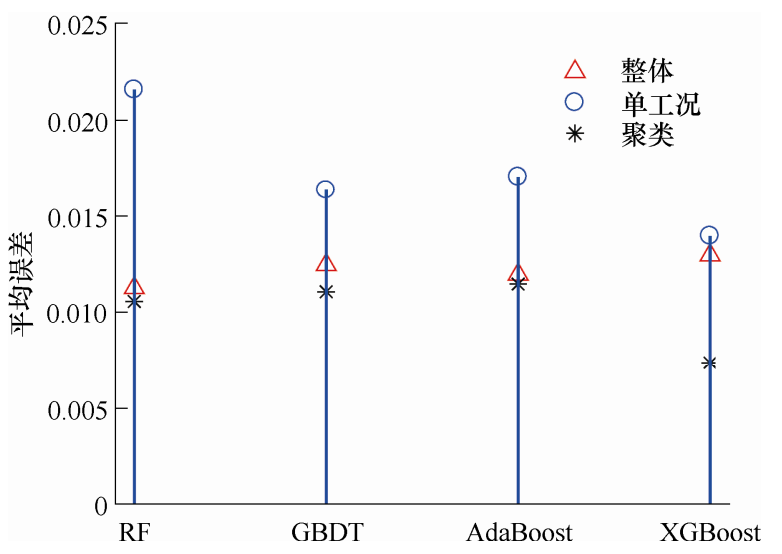

图 12 不同分类方式的平均误差对比

容易发现采用聚类的方式平均误差是最小的, 采用整体数据进行训练产生的误差会略大, 而对单 工况进行训练所产生的误差明显大于前两者。由此 说明，采用聚类的方式对工况进行分类对预测结果 有明显的改善。 


\section{2 分类预测}

采用分类的方式预测对实际应用有更为明确的 意义, 分类的第一步是确定刀具报废的标准。刀具 在磨损到一定程度之后, 就会影响尺寸精度和表面 质量, 该磨损的临界值即是刀具的磨针标准。ISO 3685 推荐的磨钝标准是: 对于硬质合金刀具, 如果 后刀面是均匀磨损, 取 $\mathrm{VB}=0.3 \mathrm{~mm}$; 如果后刀面是 不均匀磨损(存在划伤、沟痕等), 取 $\mathrm{VB}_{\max }=0.6 \mathrm{~mm}$; 本试验中定义 $V B \geqslant 0.6 \mathrm{~mm}$ 的刀具为报废刀具。

对于分类的评价标准，一般会考虑报废刀具有 多少被预测出来(召回率) 以及被预测为报废的刀具 有多少是真正的报废刀具(精确率)。精确率和召回 率是相互制约的, 为了兼顾两种评价指标, 一般取 两者的调和平均 $F_{1}$ 值作为评价指标。仍采用前述 的 4 种评估器进行训练和分类, 评估结果如图 13 所示。

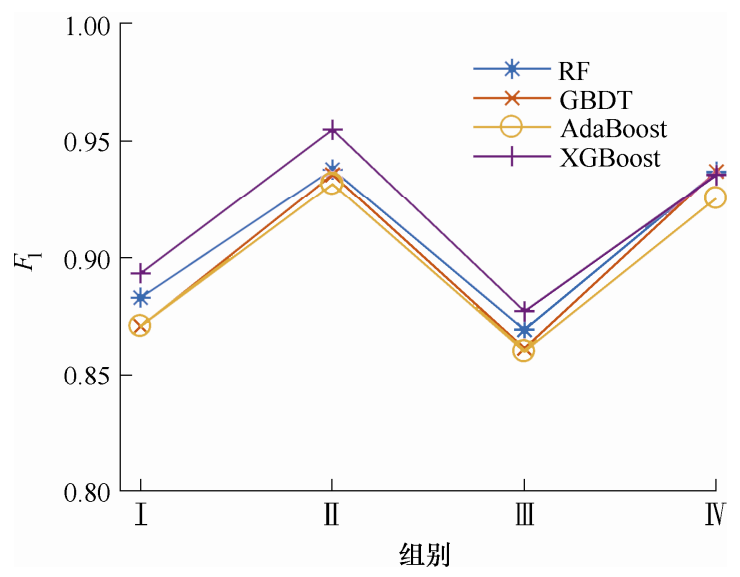

图 13 不同评估器分类 $\mathrm{F}_{1}$ 值

可以看出, 在分类问题上, 尽管各方法相差不 大，但 XGBoost 仍是结果最好的方法。

\section{3 结果对比}

在第 6.1 与 6.2 节中采用电流信号与振动信号联 合分析的方法对刀具的磨损进行了预测, 下面单独 使用电流信号或振动信号进行评估, 并和使用混合 信号的结果进行对比, 采用同样的算法(t-SNE+XGBoost) 和评估流程(回归预测和分类预测), 结果如图 14 和 图 15 所示。

可以看出, 单独使用电流信号或振动信号仍然 可以完成刀具磨损的评估, 但在精度上均有所下降, 相比之下, 振动信号比电流信号有更为优异的表现, 这是由于振动信号直接通过传感器测量获得, 实时 性好、频率高, 而电流通过内置传感器获取, 实时 性略差、频率略低。由此说明, 联合使用电流信号 与振动信号相比于单独使用其中一种信号可以在刀 具磨损评估上取得更好的效果。

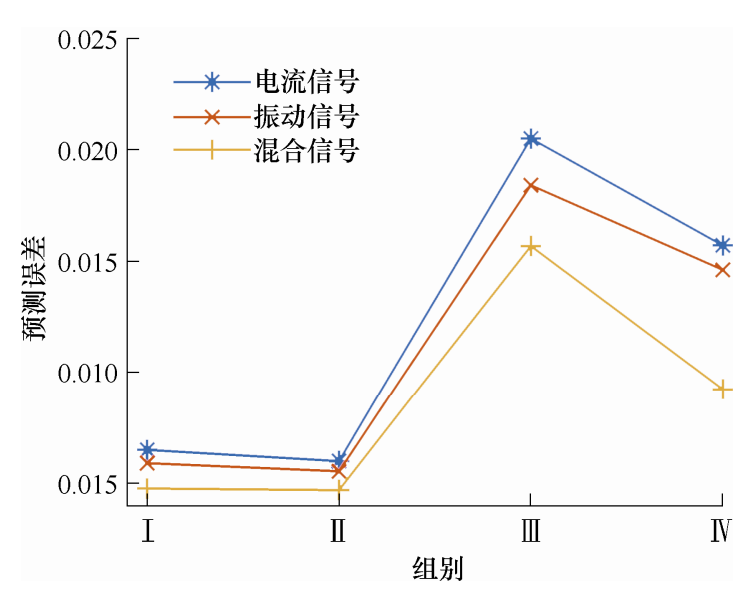

图 14 不同信号回归预测误差对比

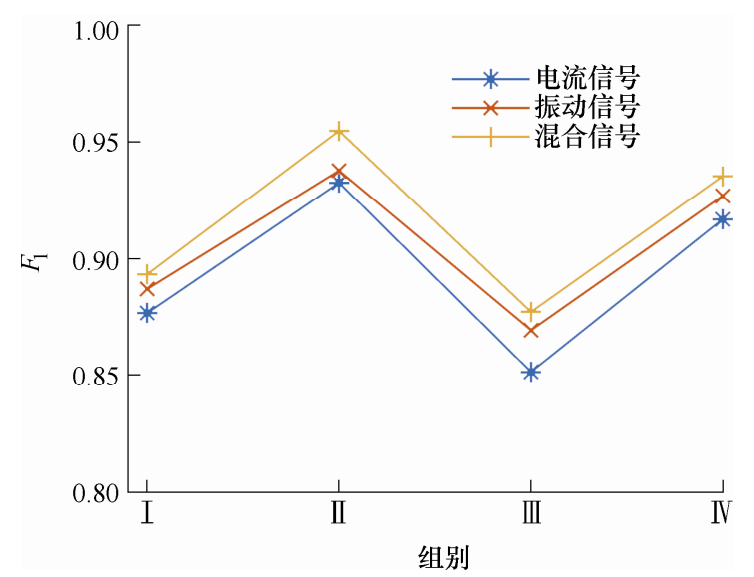

图 15 不同信号分类预测 $\mathrm{F}_{1}$ 值对比

\section{7 结论}

刀具是数控机床的关键部件, 其健康状况直接 影响到加工质量, 本文针对刀具的磨损提出了一个 完整的评估方案, 该方案综合使用了机床主轴的电 流信号与振动信号, 先是对信号进行分割, 划分出 包含有效信息的数据，然后提取时域、频域以及小 波包特征, 之后采用 t-SNE 算法对特征进行降维, 并使用 K-means 算法对工况进行聚类, 最后采用 XGBoost 评估器进行刀具磨损量的预测, 得出以下 结论。

(1) 对于刀具加工中的不同工况, 如果不进行 工况分类, 评估的准确率较低, 如果针对每一种 工况分类, 模型的泛化能力较低, 而采用工况聚 类的方式可以有效地提高模型预测的准确率和泛 化能力。

(2) 对于刀具加工所产生的信号特征, 由于刀 具磨损量的变化, 采用传统的降维方式很容易产生 信号间的混淆, 而采用 t-SNE 降维方式可以通过概 率映射有效地区分不同工况。

(3) 刀具本身磨损的特性会导致样本的分布不 
平衡, 采用集成学习的方法可以有效地处理这类问 题。而在主流的集成学习算法中, XGBoost 相较其 它算法在精度上有明显提高, 而在计算时间上可以 减少一个数量级, 是一种准确高效的预测方法。

（4）本文试验采用的是同种工件材质和工序, 在 之后的研究中可以针对不同材质的工件和不同的工 序进行研究, 探索复杂工业环境下模型的评估能力。

\section{参 考 文 献}

[1] KURADA S, BRADLEY C. A review of machine vision sensors for tool condition monitoring[J]. Computers in Industry, 1997, 34(1): 55-72.

[2] SU J C, HUANG C K, TARNG Y S. An automated flank wear measurement of microdrills using machine vision[J]. Journal of Materials Processing Technology, 2006, 180(1): 328-335.

[3] LI L, AN Q. An in-depth study of tool wear monitoring technique based on image segmentation and texture analysis[J]. Measurement, 2016, 79(2): 44-52.

[4] ANANDAKRISHNAN V, MAHAMANI A. Investigations of flank wear, cutting force, and surface roughness in the machining of Al-6061-TiB 2 in situ metal matrix composites produced by flux-assisted synthesis[J]. International Journal of Advanced Manufacturing Technology, 2011, 55(1-4): 65-73.

[5] RAO K V, MURTHY B S N, RAO N M. Prediction of cutting tool wear, surface roughness and vibration of work piece in boring of AISI 316 steel with artificial neural network[J]. Measurement, 2014，51(1): 63-70.

[6] 王丽华, 杨家嵬, 张永宏, 等. 基于堆叠降噪自编码的 刀具磨损状态识别[J]. 中国机械工程, 2018, 29(17): 2038-2045.

WANG Lihua , YANG Jiawei, ZHANG Yonghong, et al. Tool wear condition recognition based on SDAE[J]. China Mechanical Engineering ， 2018 ， 29(17): 2038-2045.
[7] NIAKI F A, ULUTAN D, MEARS L. In-process tool flank wear estimation in machining gamma-prime strengthened alloys using Kalman filter [J]. Procedia Manufacturing, 2015, 1: 696-707.

[8] KADU R S, AWARI G K, SAKHALE C N, et al. Formulation of mathematical model for the investigation of tool wears in boring machining operation on cast iron using carbide and CBN tools[J]. Procedia Materials Science, 2014, 6(1): 1710-1724.

[9] MAATEN L V D, HINTON G. Visualizing data using t-SNE[J]. Journal of Machine Learning Research, 2008, 9(2605): 2579-2605.

[10] CHEN T, GUESTRIN C. XGBoost: A scalable tree boosting system[C]// Proceedings of the Acm Sigkdd International Conference on Knowledge Discovery and Data Mining. ACM, 2016: 785-794.

[11] 赵帅, 黄亦翔, 王浩任, 等. 基于随机森林与主成分分 析的刀具磨损评估 [J]. 机械工程学报, 2017，53(21): 181-189.

ZHAO Shuai, HUANG Yixiang, WANG Haoren, et al. Tool wear assessment based on random forest and principal component analysis[J]. Journal of Mechanical Engineering, 2017, 53(21): 181-189.

[12] 张康, 黄亦翔, 赵帅, 等. 基于 $\mathrm{t}-\mathrm{SNE}$ 数据驱动模型的 盾构装备刀盘健康评估 [J]. 机械工程学报, 2019, 55(7): 19-26.

ZHANG Kang, HUANG Yixiang, ZHAO Shuai, et al. Health assessment of shield equipment based on t-SNE data driven model[J]. Journal of Mechanical Engineering, 2019, 55(7): 19-26.

作者简介: 李亚, 男, 1994 年出生。主要研究方向为数控机床智能维护。 E-mail: ly9842@sjtu.edu.cn 黄亦翔(通信作者), 男, 1980 年出生, 博士, 讲师。主要研究方向为机 电系统智能维护、模式识别、特征提取与降维。

E-mail: huang.yixiang@sjtu.edu.cn 